\title{
Effect of polydispersity and soft interactions on the nematic versus smectic phase stability in platelet suspensions
}

\author{
Y. Martínez-Ratón ${ }^{1, a)}$ and E. Velasco² \\ ${ }^{1}$ Grupo Interdisciplinar de Sistemas Complejos (GISC), Departamento de Matemáticas, Escuela Politécnica \\ Superior, Universidad Carlos III de Madrid, Avenida de la Universidad 30, E-28911 Leganés, Madrid, Spain \\ ${ }^{2}$ Departamento de Física Teórica de la Materia Condensada and Instituto de Ciencia de Materiales Nicolás \\ Cabrera, Universidad Autónoma de Madrid, E-28049 Madrid, Spain
}

(Received 31 January 2011; accepted 7 March 2011; published online 24 March 2011)

\begin{abstract}
We theoretically discuss, using density-functional theory, the phase stability of nematic and smectic ordering in a suspension of platelets of the same thickness but with a high polydispersity in diameter, and study the influence of polydispersity on this stability. The platelets are assumed to interact like hard objects, but additional soft attractive and repulsive interactions, meant to represent the effect of depletion interactions due to the addition of nonabsorbing polymer, or of screened Coulomb interactions between charged platelets in an aqueous solvent, respectively, are also considered. The aspect (diameter-to-thickness) ratio is taken to be very high, in order to model solutions of mineral platelets recently explored experimentally. In this regime a high degree of orientational ordering occurs; therefore, the model platelets can be taken as completely parallel and are amenable to analysis via a fundamental-measure theory. Our focus is on the nematic versus smectic phase interplay, since a high degree of polydispersity in diameter suppresses the formation of the columnar phase. When interactions are purely hard, the theory predicts a continuous nematic-to-smectic transition, regardless of the degree of diameter polydispersity. However, polydispersity enhances the stability of the smectic phase against the nematic phase. Predictions for the case where an additional soft interaction is added are obtained using mean-field perturbation theory. In the case of the one-component fluid, the transition remains continuous for repulsive forces, and the smectic phase becomes more stable as the range of the interaction is decreased. The opposite behavior with respect to the range is observed for attractive forces, and in fact the transition becomes of first order below a tricritical point. Also, for attractive interactions, nematic demixing appears, with an associated critical point. When platelet polydispersity is introduced the tricritical temperature shifts to very high values. (C) 2011 American Institute of Physics. [doi:10.1063/1.3570964]
\end{abstract}

\section{INTRODUCTION}

The issue of polydispersity is crucial to understand phase behavior in experiments on colloidal suspensions of spherical and anisometric particles. The latter, of either rodlike or platelike shape, are known to form liquid-crystalline phases $^{1-8}$ and their phase equilibria are largely affected by size dispersity. ${ }^{9-11}$ Colloidal particles can never be made truly identical, and even a small amount of polydispersity is unavoidable in real samples.

Platelets with varying degrees of polydispersity, in particular, are being extensively studied in recent years. In this material, polydispersity greatly facilitates gelation, and in fact the nematic phase is difficult to reach in suspensions of charge-stabilized platelike particles (the most common platelike particles) at particle concentrations below that at which a glassy state is formed. ${ }^{12}$ However, it was possible to obtain a nematic phase in hardlike platelets, e.g., gibbsite particles with steric stabilizers. ${ }^{6}$ It has been demonstrated that the observation of equilibrium nematic phases in suspensions of charged colloidal platelets requires fine-tuning of the ionic

\footnotetext{
a) Author to whom correspondence should be addressed. Electronic mail: yuri@math.uc3m.es.
}

strength of the solvent. ${ }^{6,13}$ Therefore, the concomitant effects of polydispersity and soft interactions seem to be an important issue as regards the stabilization of the nematic phase.

Besides the nematic phase, liquid-crystalline phases with partial spatial order in colloidal suspensions of platelets are now being investigated. ${ }^{14}$ The most common phase is the columnar phase, which admits a surprisingly large (up to $25 \%$ ) degree of polydispersity in diameter. Suspensions of sterically stabilized gibbsite platelets with added nonabsorbing polymer exhibit a rich phase diagram, with gravityinduced three-phase coexistence regions involving isotropic, nematic, and columnar phases, and enhanced fractionation effects. ${ }^{15}$ By contrast, observations of the layered smectic phase are very rare in these systems, ${ }^{16-18}$ since the columnar phase is very stable at high particle concentrations.

In a recent paper, ${ }^{18}$ smectic ordering was observed in colloidal suspensions of equally thick charged platelets with high polydispersity in diameter and in a solvent with low ionic strength. It is thought that the extreme diameter polydispersity ( $\gtrsim 30 \%$ ), possibly combined with the monodispersity in thickness, is responsible for the instability of the columnar phase in favor of the smectic phase. It would be interesting to theoretically analyze the effect of polydispersity on the 
formation of the spatially ordered smectic phase in systems where the columnar phase is suppressed.

On the other hand, additional soft interactions are known to affect the phase diagram. These interactions can be screened Coulomb particle interactions due to the addition of salt to an aqueous solution of charged particles, or attractive depletion forces ${ }^{19}$ between the colloidal particles arising from addition of nonabsorbing polymer to a suspension of effectively hard platelets. ${ }^{20-23}$ The possibility of theoretically predicting how both factors (polydipersity and soft interactions) influence the formation of phases with partial spatial order (smectic and columnar phases) would be highly desirable.

In the present paper we study the effects of polydispersity and soft interactions on the nematic versus smectic phase equilibrium. The work was inspired by the paper by Sun and co-workers, ${ }^{18}$ who synthesized novel platelet particles from $\alpha$-ZrP minerals by a special exfoliation procedure, which creates a perfectly monodisperse thickness distribution ${ }^{24,25}$ with high polydispersity in diameter. Low ionic-strength aqueous solutions of these charged particles form equilibrium nematic phases, followed by an incipient smectic ordering which equilibrates very slowly; no hint of columnar ordering was found. ${ }^{18}$

In the experiments by Sun $\mathrm{et} \mathrm{al.}{ }^{18}$ the ionic strength of the solution was not controlled. However, addition of salt to the suspension will modify the long-ranged repulsive interactions between platelets. Our second aim is to predict how the nematic versus smectic phase equilibrium of the suspension will be modified when the range of interactions is changed. These results could also apply to suspensions of colloidal platelets with nonabsorbing polymer, where effectively attractive depletion force between platelets usually results in demixing between phases with different concentrations of colloids and polymers $;{ }^{26}$ therefore, we also consider attractive soft interactions of varying range. As shown in Ref. 27, addition of nonabsorbing polymer to a one-component fluid of hard rods may give rise to isotropic liquid-liquid phase separation ending in a critical point (for large polymer coils), and to the presence of a broad coexistence region of isotropic-nematic, isotropicsmectic, and isotropic-crystal phases. Binary mixtures of colloidal disks and polymers exhibit not only isotropic-isotropic demixing, but also nematic-nematic demixing. ${ }^{28}$ However, nonuniform phases were not taken into account in the latter study. As will be shown below, soft interactions between highly oriented platelets can induce nematic-nematic demixing and a dramatic broadening of the nematic-smectic (NS) transition region for sufficiently long-ranged interactions.

The effect of polydispersity on the phase behavior of hard rods has been theoretically studied at the level of density-functional theory (using the Onsager second virial theory) mainly for uniform liquid crystal phases (isotropic or nematic). ${ }^{29-31}$ Also, a bimodal polydisperse distribution function in aspect ratio for hard boardlike particles was used to study the effect of polydispersity on the phase stability of biaxial nematic versus nematic-nematic demixing. ${ }^{32,33}$ The limit of zero polydispersity gives rise to a perfect binary (bidisperse) mixture, a case studied by Varga and coworkers ${ }^{34}$ using the Onsager theory. Harnau et al..$^{35}$ and Bier et al. ${ }^{36}$ have also studied perfect binary mixtures of platelets

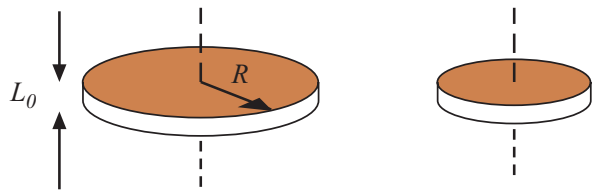

FIG. 1. Schematic of the parallel cylindrical particles used in this work.

using density-functional theory ${ }^{36}$ in the restricted-orientation approximation. Finally, a simulation study was done by Bates and Frenkel on the influence of polydispersity on nonuniform phases of rodlike particles: it was shown ${ }^{10}$ that the smectic phase of hard colloidal rods can be destabilized with respect to the columnar phase for high enough length polydispersity.

Density-functional studies of the relative stability of nonuniform (smectic, columnar, and crystal) phases in polydisperse fluids made of anisotropic particles constitute a great challenge. The main difficulty is related to the numerical minimization of the functional as the density profile is a function of the spatial, angular, and polydisperse variables. The present work is a first attempt to tackle the study of smectic phases in diameter-polydisperse platelets using density-functional tools and, in particular, fundamental-measure theory (FMT) of parallel hard cylinders. ${ }^{37}$

We first give a brief overview of the theory in Sec. II. In Sec. III we examine the influence of polydispersity on the phase behavior of hard parallel platelets, while the case of soft platelets is treated in Sec. IV. We end with some conclusions in Sec. V.

\section{MODEL AND THEORY}

The particle model consists of cylinders with parallel axes of revolution (Fig. 1). This approximation is justified in the regime of high packing fractions where the NS transition occurs (obviously the isotropic-nematic transition cannot be treated within this scheme, but this is not the aim of the present study, which is focused on the high-density regime, where the isotropic phase is not stable). Cylinders have the same thickness, $L_{0}$, in line with the particles obtained by Sun et al. ${ }^{18}$ but are polydisperse in diameter. We assume the fluid to consist of a multicomponent mixture of species with different radii $R$. In the thermodynamic limit the fluid contains infinitely many species, and polydispersity can be characterized by means of a continuous size distribution. The particle radius will be characterized by the scaled variable $r=R / R_{0}$, with $R_{0}$ the mean radius. The number fraction of a given species with radius $r$ will be $p(r)$, the (continuous) radius distribution to be specified later.

\section{A. Density-functional theory}

The statistical mechanics of a fluid of $N$ parallel cylindrical platelets in a volume $V$ will be investigated using the FMT density-functional approach for a multicomponent mixture of parallel cylinders presented in Ref. 37. In that work, the excess part of the free-energy density functional of a mixture of parallel hard cylinders was obtained from the corresponding 
functional for a multicomponent mixture of hard disks by applying the dimensional cross-over criterion. ${ }^{37}$ This criterion uses the fact that, when the cylinders are parallel and their centers of mass are constrained to be on a plane, the free energy of the original system reduces to that of a mixture of hard disks (the cylinder cross sections). In turn, the density functional of a hard-disk mixture can be obtained from the knowledge of (i) its low-density limit (the second virial approach) and (ii) its zero-dimensional limit (i.e., when the density profile is consistent with that of a cavity that can only accommodate one particle at most). As shown in Ref. 38, the obtained density functional for parallel hard cylinders predicts equations of state for the nematic, smectic, and crystalline phases in very good agreement with simulation results of the onecomponent system.

The free-energy density obtained by this approach is a function of certain one- or two-body weighted densities that can be obtained by convoluting the density profiles of different species with some weighting functions and then summing over all species. For the case of smectic symmetry, the twobody weighted densities decouple into a product of two onebody densities. ${ }^{38}$ Here, we trivially extend this approach to the polydisperse case by substituting these sums over species by integrals with respect to the polydisperse variable (the diameter of platelets).

We choose the cylinder axes, the nematic director, and the layer normal in the smectic phase to be parallel and along the $z$ direction. In the density-functional approach the central quantity is the number density $\rho(z, r)$, which gives the local density at $z$ of the species with radius $r$ and satisfies

$$
\int_{0}^{\infty} d r \int_{V} d \mathbf{r} \rho(z, r)=N .
$$

Note that $\mathbf{r}=(x, y, z)$ refers to the space coordinate vector, while $r$ is the scaled radius of particle (not the modulus of r). The excess part of the local free-energy density (assuming smectic symmetry) is (see Refs. 37 and 38 for more details)

$$
\begin{aligned}
\Phi(z) \equiv \beta f_{\mathrm{ex}}(z)= & -n_{0}\left(1-n_{3}\right)+\frac{2 n_{1}^{\perp} n_{2}^{\perp}+n_{1}^{\|} n_{2}^{\|}}{1-n_{3}} \\
& +\frac{n_{2}^{\|}\left(n_{2}^{\perp}\right)^{2}}{\left(1-n_{3}\right)^{2}},
\end{aligned}
$$

where $f_{\text {ex }}$ is the local excess free-energy density, $\beta=1 / k T$, and $k$ is Bolztmann's constant, with $T$ the temperature. The one-body weighted densities $n_{0}(z), n_{1}^{\|}(z), n_{1}^{\perp}(z), n_{2}^{\|}(z), n_{2}^{\perp}(z)$, and $n_{3}(z)$ [in Eq. (2) their $z$ dependence has not been explicitly indicated] are obtained from the number density $\rho(z, r)$ using the expressions

$$
\begin{aligned}
& n_{0}(z)=\left[\mathcal{M}_{0} * \omega^{(0)}\right](z), \quad n_{1}^{\|}(z)=\pi\left[\mathcal{M}_{0} * \omega^{(3)}\right](z), \\
& n_{1}^{\perp}(z)=\left[\mathcal{M}_{1} * \omega^{(3)}\right](z), \quad n_{2}^{\|}(z)=\left[\mathcal{M}_{2} * \omega^{(0)}\right](z), \\
& n_{2}^{\perp}(z)=\pi\left[\mathcal{M}_{1} * \omega^{(3)}\right](z), \quad n_{3}(z)=\pi\left[\mathcal{M}_{2} * \omega^{(3)}\right](z),
\end{aligned}
$$

where the symbol $*$ stands for convolution. The functions

$$
\mathcal{M}_{\alpha}(z)=\int_{0}^{\infty} d r \rho(z, r) R^{\alpha}
$$

are generalized moments of the polydispersity distribution, while

$$
\omega^{(0)}(z)=\frac{1}{2} \delta\left(\frac{L_{0}}{2}-|z|\right), \quad \omega^{(3)}(z)=\Theta\left(\frac{L_{0}}{2}-|z|\right),
$$

with $\delta$ and $\Theta$ the Dirac delta and Heaviside functions, respectively. The excess free-energy functional is obtained by integration over the system volume $V$, i.e., $\beta \mathcal{F}_{\text {ex }}[\rho]=\int_{V} d \mathbf{r} \Phi(z)$. Adding the ideal contribution $\beta \mathcal{F}_{\text {id }}[\rho]$, with

$$
\beta \mathcal{F}_{\text {id }}[\rho]=\int_{0}^{\infty} d r \int_{V} d \mathbf{r} \rho(z, r)\left\{\log \left[\rho(z, r) \Lambda_{r^{3}}\right]-1\right\},
$$

where $\Lambda_{r}$ is the thermal wavelength of the species with radius $r$, we obtain the total free-energy functional as $\mathcal{F}[\rho]$ $=\mathcal{F}_{\text {id }}[\rho]+\mathcal{F}_{\text {ex }}[\rho]$. The equilibrium state of the system follows from functional minimization of $\mathcal{F}[\rho]$ with respect to the local number densities $\rho(z, r)$.

The radius distribution function $p(r)$ was chosen to be a Schultz distribution:

$$
p(r)=\frac{(v+1)^{v+1}}{\Gamma(v+1)} r^{v} \exp [-(v+1) r],
$$

where $v$ is a free parameter that controls the width of the distribution. We have checked that our results are not qualitatively sensitive to any particular function $p(r)$, and that the only requirement to be imposed on $p(r)$ is the existence of well-defined moments, i.e., the moments have to always be finite. Once $p(r)$ is chosen, the local density $\rho(z, r)$ must satisfy the normalization relation

$$
p(r)=\frac{\int_{V} d \mathbf{r} \rho(z, r)}{\int_{V} d \mathbf{r} \int_{0}^{\infty} d r \rho(z, r)}=\frac{1}{N} \int_{V} d \mathbf{r} \rho(z, r) .
$$

Defining the moments as

$$
m_{\alpha}=\int_{0}^{\infty} d r p(r) r^{\alpha},
$$

the distribution (7) fulfills $m_{0}=m_{1}=1$. The polydispersity coefficient $\Delta$, defined as usual as the width of the radius distribution, $\Delta=\left(m_{2}-m_{1}^{2}\right)^{1 / 2} / m_{1}$, is related to $v$ by $\Delta=(v+1)^{-1 / 2}$. In the smectic phase, local moments $m_{\alpha}(z)$ of the density distribution can also be defined:

$$
m_{\alpha}(z)=\frac{1}{\rho_{0}} \int_{0}^{\infty} d r r^{\alpha} \rho(z, r),
$$

where $\rho_{0}=N / V$ is the mean density. Obviously

$$
\frac{1}{V} \int_{V} d \mathbf{r} m_{\alpha}(z)=\frac{1}{d} \int_{0}^{d} d z m_{\alpha}(z)=m_{\alpha},
$$

where $d$ is the smectic period. 


\section{HARD PLATELETS POLYDISPERSE IN DIAMETER}

In the one-component case (platelets of the same diameter), the NS transition is located at a value of packing fraction $\eta_{s}^{(0)}=0.314,{ }^{38}$ where the packing fraction is defined as $\eta=\rho_{0} v$, with $v=\pi R_{0}^{2} L_{0}$ the mean particle volume and $\rho_{0}=N / V$ the mean density. The transition is continuous. The question we would like to answer is whether the transition is still continuous when polydispersity is introduced and how the packing fraction at the transition behaves as the sample polydispersity is changed.

\section{A. Nematic-smectic bifurcation}

As a preliminary step prior to the full minimization of the functional, we have calculated the spinodal (or bifurcation) line for the NS transition of the fluid. This can give us an indication of the basic trends. In particular, if the transition is of the second order, the bifurcation line will coincide with the transition line. In case the transition is of the first order, coexistence conditions, and therefore a full functional minimization that can furnish the pressure and chemical potentials, will be necessary (Sec. III B).

The calculation starts by perturbing the nematic with a small-amplitude density wave, $\rho(z, r)=\rho_{0} p(r)$ $+\epsilon(r) \cos k z$, where $k=2 \pi / d$ is a wavenumber. The fluid response to the perturbation is obtained from the Fourier transform of the direct correlation function (taken from the second functional derivative of the excess free-energy functional):

$$
\begin{aligned}
-\rho_{0} \hat{c}\left(k ; r, r^{\prime}\right)= & c_{0}(k)\left(r+r^{\prime}\right)^{2}+c_{1}(k) r r^{\prime}\left(r^{2}+r^{\prime 2}\right) \\
& +c_{2}(k)\left(r r^{\prime}\right)^{2},
\end{aligned}
$$

where $r, r^{\prime}$ are the scaled radii of two platelets. Here we have defined the coefficients

$$
\begin{aligned}
c_{0}(k)= & y\left[2 \chi(k)+y m_{2} \chi^{2}\left(\frac{k}{2}\right)\right], \\
c_{1}(k)= & 2 y^{2}\left[2 \chi(k)+\left(1+2 y m_{2}\right) \chi^{2}\left(\frac{k}{2}\right)\right], \\
c_{2}(k)= & y^{2}[2(1+2 y) \chi(k) \\
& \left.+\left(1+2 y\left(2+m_{2}\right)+6 m_{2} y^{2}\right) \chi^{2}\left(\frac{k}{2}\right)\right],
\end{aligned}
$$

where $\chi(k)=\sin k^{*} / k^{*}$ (with $k^{*}=k L_{0}$ the scaled wavenumber) and $y=\eta /\left(1-\eta m_{2}\right)$. The instability of the fluid against the density wave is obtained from the eigenvalue problem

$$
\epsilon(r)=\rho_{0} p(r) \int d r^{\prime} \hat{c}\left(k, r, r^{\prime}\right) \epsilon\left(r^{\prime}\right),
$$

from which the wavenumber $k_{s}$ and packing fraction $\eta_{s}$ at bifurcation can be obtained.

Figure 2 shows the packing fraction of the NS spinodal, $\eta_{s}$, relative to that of the monodisperse fluid, $\eta_{s}^{(0)}$, as a function of polydispersity $\Delta$. The inset shows the radius distribution function for the particular value $\Delta=0.6$. The packing fraction at bifurcation decreases as particles become more polydisperse in radius, so the smectic phase becomes more stable with respect to the nematic with increasing polydis-

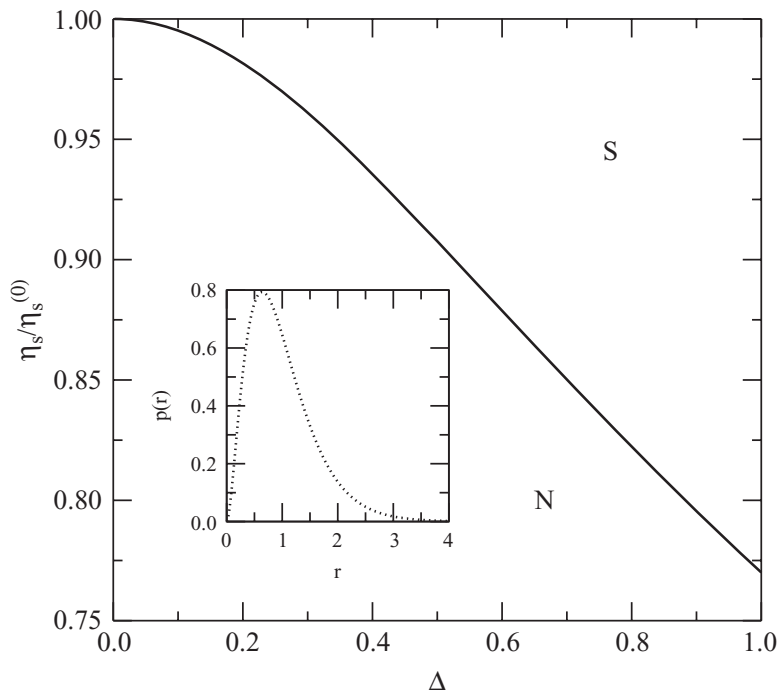

FIG. 2. Packing fraction $\eta_{s}$ at the NS spinodal, relative to that of the monodisperse fluid $\eta_{s}^{(0)}$, as a function of polydispersity parameter $\Delta$ for hard platelets. Labels indicate the nematic $(\mathrm{N})$ and smectic $(\mathrm{S})$ regions. The inset is the radius distribution function at the spinodal for $\Delta=0.6$.

persity. This trend is opposite to that observed in fluids of hard rods, where polydispersity in length postpones the onset of smectic stability due to incommensurability of the particle lengths with a smectic period.

\section{B. Full minimization and search for coexistence}

A full minimization of the functional is required in order to gain more insight into the true nature (i.e., continuous versus first order) of the NS transition and to obtain information on the equation of state, smectic period, and possible fractionation effects in the case $\Delta>0$ (i.e., the fact that the radius distribution function can be different in the two coexisting phases).

We wish to calculate the density profile $\rho(z, r)$ of a stable smectic phase with a fixed number density $\rho_{0}$ and a prescribed radius distribution function $p(r)$. Note that the nematic phase will be unstable or metastable. In the minimization of the freeenergy functional per unit volume, $\beta \mathcal{F} / V$, the density distribution is subject to the constraint $\rho_{0} p(r)=\langle\rho(z, r)\rangle_{d}$ [see Eq. (8)], with the shorthand notation $\langle\cdots\rangle_{d} \equiv d^{-1} \int_{0}^{d} d z(\cdots)$ for an average over the smectic period. Direct functional minimization leads to

$$
\rho(z, r)=\frac{\rho_{0} p(r) \exp \left[c^{(1)}(z, r)\right]}{\left\langle\exp \left[c^{(1)}(z, r)\right]\right\rangle_{d}},
$$

with $c^{(1)}(z, r)$ the one-body correlation function. This function is a quadratic polynomial in $r$, with coefficients which are functionals of the moments $m_{\alpha}(z)=\rho_{0}^{-1} \int_{0}^{\infty} d r r^{\alpha} \rho(z, r)$. From Eq. (15) we obtain a set of self-consistent nonlinear integral equations in $m_{\alpha}(z)$ :

$$
m_{\alpha}(z)=\int_{0}^{\infty} d r \frac{r^{\alpha} p(r) \exp \left[c^{(1)}(z, r)\right]}{\left\langle\exp \left[c^{(1)}(z, r)\right]\right\rangle_{d}}, \quad \alpha=0,1,2 .
$$



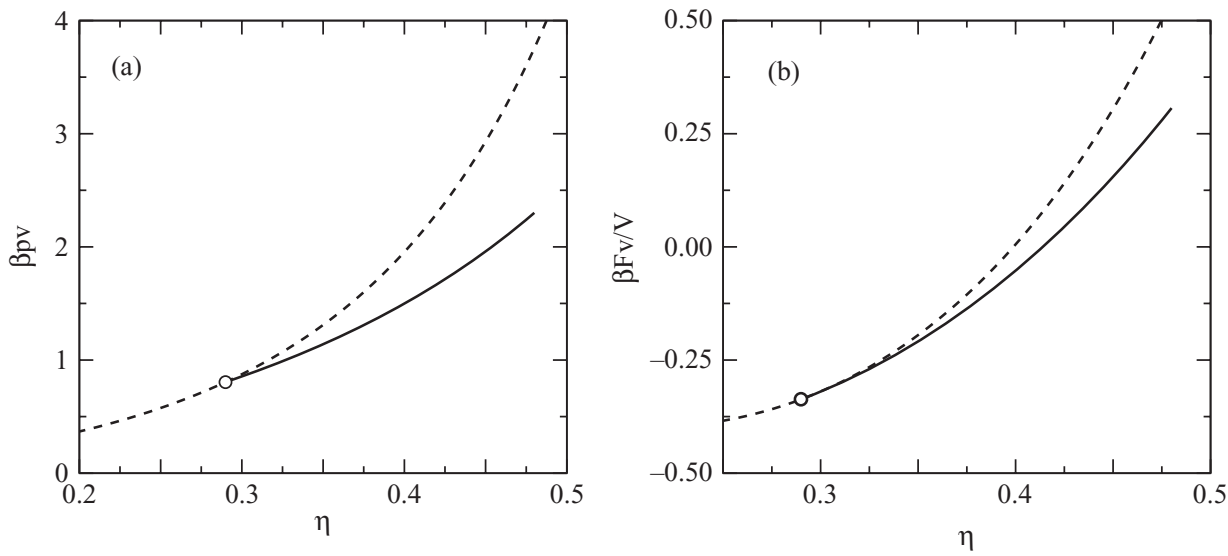

FIG. 3. (a) Scaled pressure $\beta P v$ and (b) scaled free energy $\beta \mathcal{F} v / V$ as a function of packing fraction $\eta$ for the nematic (dashed curve) and smectic (continuous curve) branches of hard polydisperse platelets. The circle indicates the location of the bifurcation point. The value of polydispersity is $\Delta=0.447$.

Expanding the local moments $m_{\alpha}(z)$ in a Fourier series containing the wavenumber $k=2 \pi / d$, Eq. (16) can be written as self-consistent equations for the corresponding expansion coefficients. The smectic period $d$ is obtained from the free energy by minimization. For high packing fraction this process gives moments $m_{\alpha}(z)$ that are not constants: this corresponds to the smectic branch. Otherwise, the nematic branch is obtained.

Next we briefly outline how the conditions for NS coexistence were obtained. In equilibrium the chemical potentials of each species should be equal in both phases: $\mu^{(i)}(r)=\mu^{(0)}(r)$ $\left(i=\mathrm{N}, \mathrm{S}\right.$ for nematic and smectic, respectively). $\mu^{(0)}(r)$ is calculated from the level-rule constraint

$$
\gamma \rho_{\mathrm{N}}(r)+(1-\gamma) \rho_{\mathrm{S}}(r)=\rho_{0} p_{0}(r),
$$

which implies the conservation of the total number of particles; $\rho_{0}$ and $p_{0}(r)$ are the number density and radius distribution function of the parent phase. $0 \leq \gamma \leq 1$ is the fraction of the total volume occupied by the $\mathrm{N}$ phase. Note that the number density of the $\mathrm{S}$ phase is $\rho_{\mathrm{S}}(r)=\left\langle\rho_{\mathrm{S}}(z, r)\right\rangle_{d}$. Using the definition for chemical potentials,

$$
\begin{gathered}
\beta \mu^{(\mathrm{N})}(r)=\frac{\delta \beta \mathcal{F}}{\delta \rho_{\mathrm{N}}(r)}=\ln \rho_{\mathrm{N}}(r)-c_{\mathrm{N}}^{(1)}(r), \\
\beta \mu^{(\mathrm{S})}(r)=\frac{\delta \beta \mathcal{F}}{\delta \rho_{\mathrm{S}}(r, z)}=\ln \rho_{\mathrm{S}}(r, z)-c_{\mathrm{S}}^{(1)}(r, z),
\end{gathered}
$$

the equilibrium conditions give

$$
\begin{aligned}
\rho_{\mathrm{N}}(r) & =\exp \left[\beta \mu^{(0)}(r)+c_{\mathrm{N}}^{(1)}(r)\right], \\
\rho_{\mathrm{S}}(r, z) & =\exp \left[\beta \mu^{(0)}(r)+c_{\mathrm{S}}^{(1)}(r, z)\right] .
\end{aligned}
$$

Inserting these expressions into the level rule (17), we obtain the following expression for the function $\mu^{(0)}(r)$ :

$$
\begin{gathered}
\exp \left[\beta \mu^{(0)}(r)\right]=\rho_{0} p_{0}(r) T(r), \\
T^{-1}(r)=\gamma \exp \left[c_{\mathrm{N}}^{(1)}(r)\right]+(1-\gamma)\left\langle\exp \left[c_{\mathrm{S}}^{(1)}(z, r)\right]\right\rangle_{d} .
\end{gathered}
$$

This, together with Eqs. (20) and (21), provides a set of equations for the moments $m_{\alpha}^{(\mathrm{N})}$ and $m_{\alpha}^{(\mathrm{S})}(z)$ :

$$
\begin{gathered}
m_{\alpha}^{(\mathrm{N})}=\int_{0}^{\infty} d r r^{\alpha} p_{0}(r) \exp \left[c_{\mathrm{N}}^{(1)}(r)\right] T(r), \\
m_{\alpha}^{(\mathrm{S})}(z)=\int_{0}^{\infty} d r r^{\alpha} p_{0}(r) \exp \left[c_{\mathrm{S}}^{(1)}(z, r)\right] T(r) .
\end{gathered}
$$

These equations, together with the condition for mechanical equilibrium, i.e., equality of pressures of both phases $P^{(\mathrm{S})}=P^{(\mathrm{N})}$ [in our model $\beta P=\left\langle\partial \Phi / \partial n_{3}\right\rangle_{d}$ ], provide equilibrium moments of each phase and the number density $\rho_{0}$ of the parent uniform phase at coexistence.

A typical feature of polydisperse systems is that the composition of the two coexisting phases varies as the phase transition takes place. In our case this means the following. Let us fix the value of $\Delta$ (the so-called dilution line). Then, as the total packing fraction $\eta$ of the parent nematic phase is increased, first an infinitesimal amount of smectic material, called shadow smectic, will appear, coexisting with the cloud (parent) nematic phase. As $\eta$ is further increased, the amount of smectic material will grow, and eventually the nematic will disappear. The opposite process occurs by starting from a parent smectic phase and decreasing $\eta$; a smectic cloud point will be reached when the first (shadow) nematic material can be observed in the sample.

The cloud-nematic and shadow-smectic coexistence curves (which are more relevant experimentally since one usually starts from a parent nematic phase) can be calculated from Eqs. (23) and (25) by setting $\gamma=1$. This results in

$$
m_{\alpha}^{(\mathrm{S})}(z)=\int_{0}^{\infty} d r r^{\alpha} p_{0}(r) e^{\Delta c^{(1)}(z, r)}, \quad \alpha=0,1,2,
$$

where $\Delta c^{(1)}(z, r)=c_{\mathrm{S}}^{(1)}(z, r)-c_{\mathrm{N}}^{(1)}(r)$ is the difference in the one-body correlation functions of the two phases. In this case the nematic radius distribution function coincides with $p_{0}(r)$ (that of the parent phase). The smectic-cloud and nematicshadow calculations can be implemented by setting $\gamma=0$ in Eqs. (23) and (24). In what follows, all coexistence results correspond to the cloud-nematic and shadow-smectic curves for different polydispersities. 


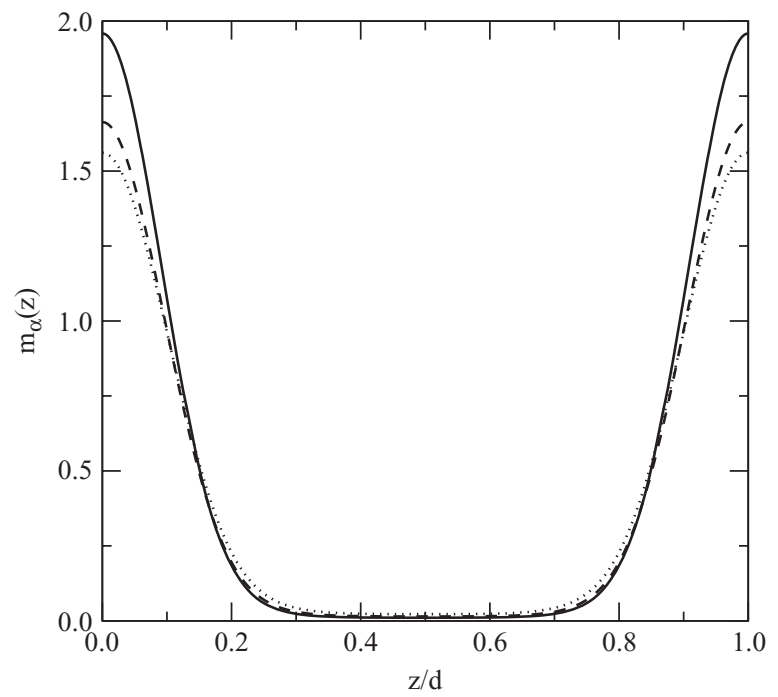

FIG. 4. Profiles of the moments $m_{\alpha}(z)$, with $\alpha=0,1,2$ (continuous, dashed, and dotted curves, respectively), along one smectic period for hard polydisperse platelets in the case $\Delta=0.333$ and $\eta=0.455$. The smectic period is $d=1.212 L_{0}$.

Our first result of the full minimization is shown in Fig. 3, where the scaled pressure $\beta P v$ and the scaled free energy $\beta F v / V$ are plotted as a function of packing fraction $\eta$ for the case $\Delta=0.447$. Calculations using many different
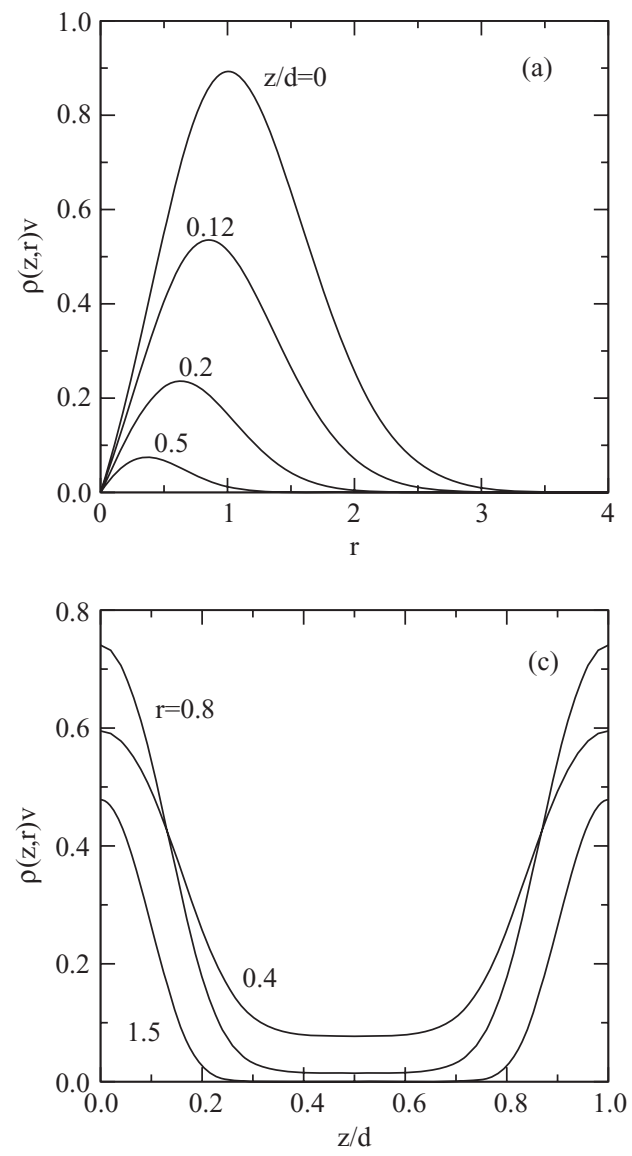

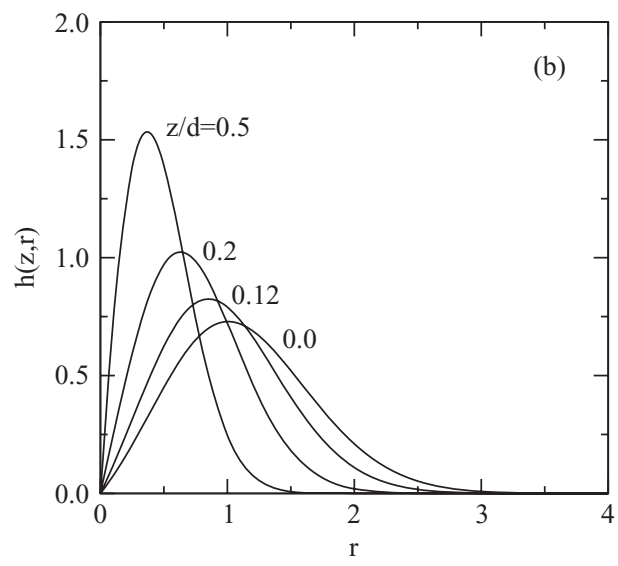

initial guesses to solve the self-consistent equations always lead to a single stable solution, which means that the nematic branch always bifurcates tangentially to a smectic branch. This implies that there is no coexistence, and that the transition is always continuous. The scenario is the same for polydispersities larger than the one used in the calculations shown in the figure.

It is instructive to examine the structure of the smectic phase by looking at the profiles of the moments $m_{\alpha}(z)$. Figure 4 plots the moments in one smectic period for the case $\Delta=0.333$ and packing fraction $\eta=0.455$, i.e., well inside the smectic region. As can be seen from the figure, the zeroth moment, $m_{0}(z)$, reflects the structure of the total density, i.e., pronounced peaks located at the smectic layers with a period of $d$. The first moment, $m_{1}(z)$, gives information about the mean particle radius at each location; at the layers the mean radius is $\simeq 1.65 R_{0}$, and it decreases away from the layers: larger particles are located preferentially at the layers, whereas smaller particles tend to stay at the interstitials.

The latter effect can be studied in more detail by analyzing the density profiles $\rho(z, r)$, along with the local radius distributions

$$
h(z, r)=\frac{\rho(z, r)}{\int_{0}^{\infty} d r \rho(z, r)},
$$

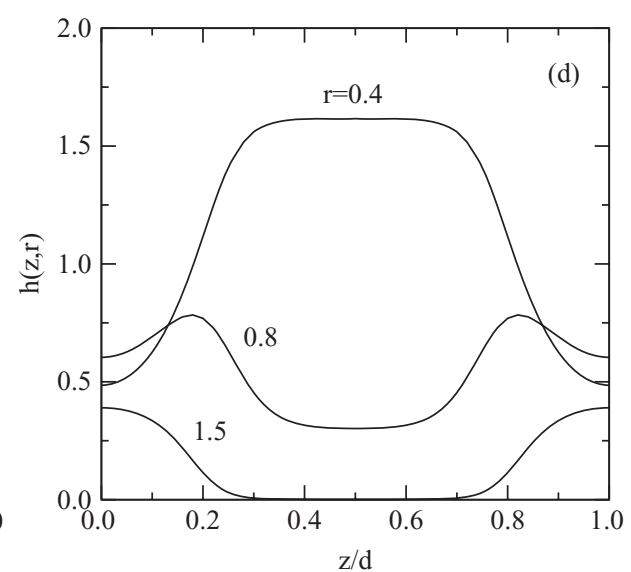

FIG. 5. Scaled density distribution $\rho(z, r) v$ and normalized density profile $h(z, r)$ for a smectic phase of polydisperse hard platelets with packing fraction $\eta=0.452$ and $\Delta=0.523$. In (a) and (b), distributions are plotted as a function of the scaled radius $r$ for particular values of the $z$ coordinate, given as labels (for reference, the smectic period is $d=1.211 L_{0}$ ). In (c) and (d), distributions are plotted as a function of the $z$ coordinate for particular values of $r$. 
as a function of $r$ for given $z$. This is shown in Figs. 5(a) and 5 (b) for four different values of $z$. Complementary plots are presented in Figs. 5(c) and 5(d), where the same functions are given as a function of $z$ for three particular values of $r$. The conclusion that can be drawn from the figures is that there exists a microsegregation effect in the smectic phase. Specifically, particles with larger radii are preferentially located at the smectic layers, i.e., at $z=0$ or $d$, and possess a wider distribution function; by contrast, at the interstitial, $z=d / 2$, the distribution function $h(z, r)$ has a maximum at smaller values of $r$ and is much more narrow. These microsegregation effects become more pronounced as the degree of polydispersity is increased.

The increased stability of the smectic phase in fluids of hard polydisperse platelets, shown in Fig. 2, is due to the larger packing efficiency (lower excluded volume) that platelets can achieve at the smectic layers when there is a continuous distribution of radii. As the distribution becomes wider, and through a microsegregation mechanism, larger packing fractions can be achieved at the quasi-2D-layers, which leads to a stabilization of the layered arrangements with respect to the uniformly distributed platelet configurations in the nematic phase. In fluids of polydisperse rods, microsegregation also explains the destabilization of the smectic phase, since particles of different lengths cannot be arranged favorably into identical layers because of inefficient packing, and the minority species is expelled to the interstitials.

\section{SOFT PLATELETS}

Next we consider hard platelets that interact via an additional soft potential. The soft potential will be treated by means of the usual mean-field approximation, neglecting correlations. First we treat the case of a monodisperse fluid, leaving the general case of a polydisperse fluid for Sec. IV B.

\section{A. Monodisperse fluid}

The soft potential should reflect the anisotropies of particle interactions. We have chosen a functional form corresponding to a modified Yukawa potential where transverse and longitudinal coordinates are scaled with the particle sizes $R$ and $L_{0}$, respectively:

$$
V_{\mathrm{soft}}\left(r_{\perp}, z\right)=\mp \epsilon \begin{cases}\frac{e^{-\lambda\left[\sqrt{\left(\frac{r_{\perp}}{2 R}\right)^{2}+\left(\frac{z}{L_{0}}\right)^{2}}-1\right]}}{\sqrt{\left(\frac{r_{\perp}}{2 R}\right)^{2}+\left(\frac{z}{L_{0}}\right)^{2}},} & |z|>L_{0}, \\ 0, & \text { or } \\ & |z|<L_{0} \text { and } r_{\perp}>2 R, \\ \text { otherwise, }\end{cases}
$$

where $\epsilon>0$. Here $r_{\perp}$ is the interparticle relative distance (measured from the platelet centers) in the $x y$ plane, $z$ the relative distance along $z$, and $\lambda$ an inverse interaction range parameter. Scaling the coordinates as in Eq. (28) is a natural choice, as it produces ellipsoidal-like equipotential surfaces and discriminates between face-to-face and side-by-side configuration of two platelets, which should have very different interparticle forces.

The effect of the soft interaction on the free-energy functional is obtained from perturbation theory in the mean-field approximation:

$$
\frac{\beta \mathcal{F}_{\mathrm{mf}}}{V}=\frac{1}{2 d} \int_{0}^{d} d z \int_{-\infty}^{\infty} d z^{\prime} \rho(z) \rho\left(z^{\prime}\right) u\left(\left|z^{\prime}-z\right|\right)
$$

with the effective potential

$$
u(z)=2 \pi \int_{0}^{\infty} d r_{\perp} r_{\perp} \beta V_{\text {soft }}\left(r_{\perp}, z\right) .
$$

We use a Gaussian parametrization for the smectic density profile:

$$
\rho(z)=\rho d\left(\frac{\alpha}{\pi}\right)^{1 / 2} \sum_{n} \exp \left[-\alpha(z-n d)^{2}\right],
$$

where $n$ is the set of integer values: $0, \pm 1, \pm 2, \ldots$.

In the present case of monodisperse particles, the procedure outlined in Sec. III B for obtaining the equilibrium structure of the fluid can be made much simpler. Thus, for given values of scaled temperature $T^{*}=k T / \epsilon$ and interaction range parameter $\lambda$, the calculation proceeds by fixing the value of the packing fraction $\eta$, and then minimizing the free energy with respect to the Gaussian width $\alpha$ and the smectic period $d$ to find the equilibrium density profile. Having obtained the free-energy branches as a function of $\eta$ for both nematic and smectic phases, the double tangent construction is used to find the coexisting packing fractions. Changing $T$ and repeating the whole process, the phase diagram of the model in the $T-\eta$ plane is obtained. Considering the cases or + in Eq. (28), one can cover both attractive and repulsive interactions. 

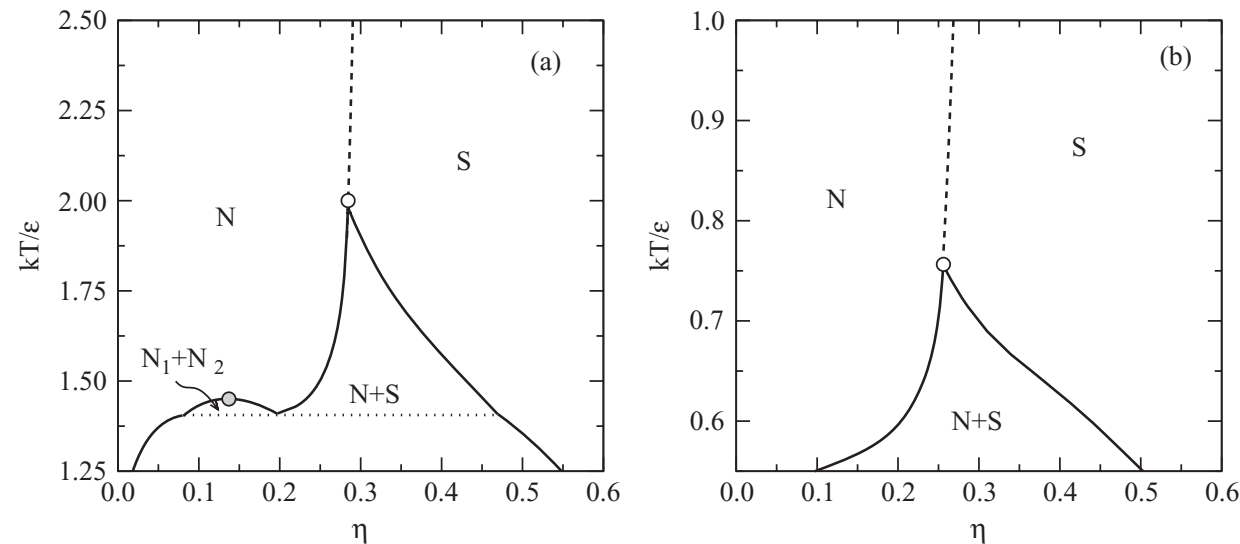

FIG. 6. Phase diagram of the monodisperse attractive Yukawa fluid. Continuous curves: coexistence boundaries for the nematic-smectic or nematic-nematic transitions. Dashed curves: nematic-smectic spinodal. Open circles: tricritical points. Shaded circle: critical point. Dotted horizontal line: tie line of triple-point $\mathrm{N}_{1}+\mathrm{N}_{2}+\mathrm{S}$ coexistence. (a) $\lambda=1$. (b) $\lambda=2$.
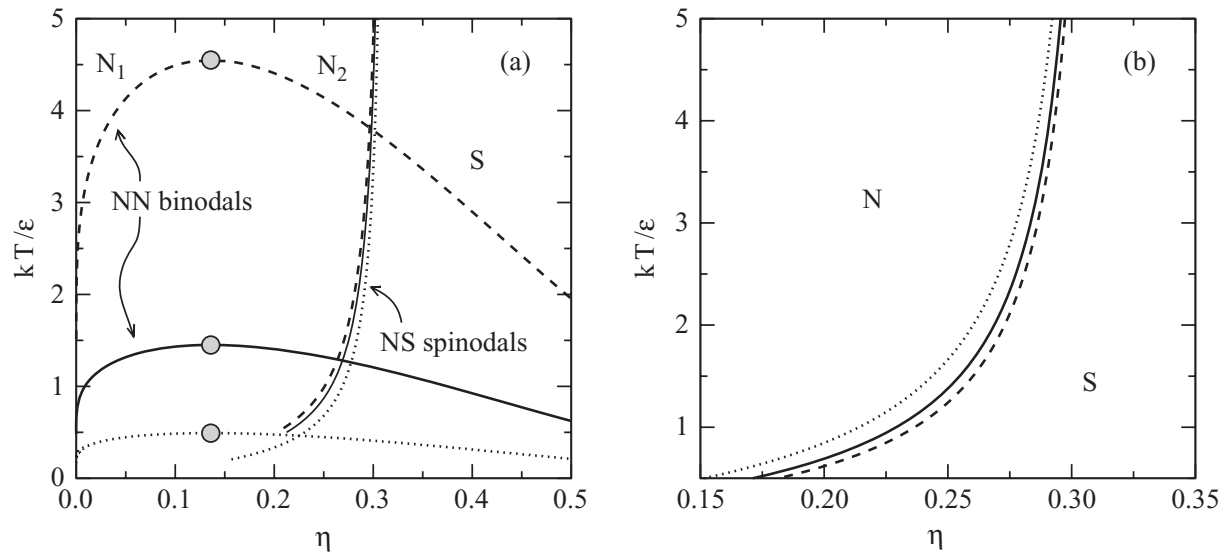

FIG. 7. Scaled-temperature-packing-fraction $T^{*}-\eta$ phase diagrams of the monodisperse model with (a) attractive and (b) repulsive soft interactions. Nematic $(\mathrm{N})$ and smectic (S) phases are labeled by symbols. In the case of demixing, two nematic phases, $\mathrm{N}_{1}$ and $\mathrm{N}_{2}$, coexist below a critical point, indicated by circles. In both panels the inverse interaction lengths are $\lambda=0.5$ (dashed curves), 1.0 (continuous curves), and 2.0 (dotted curves).
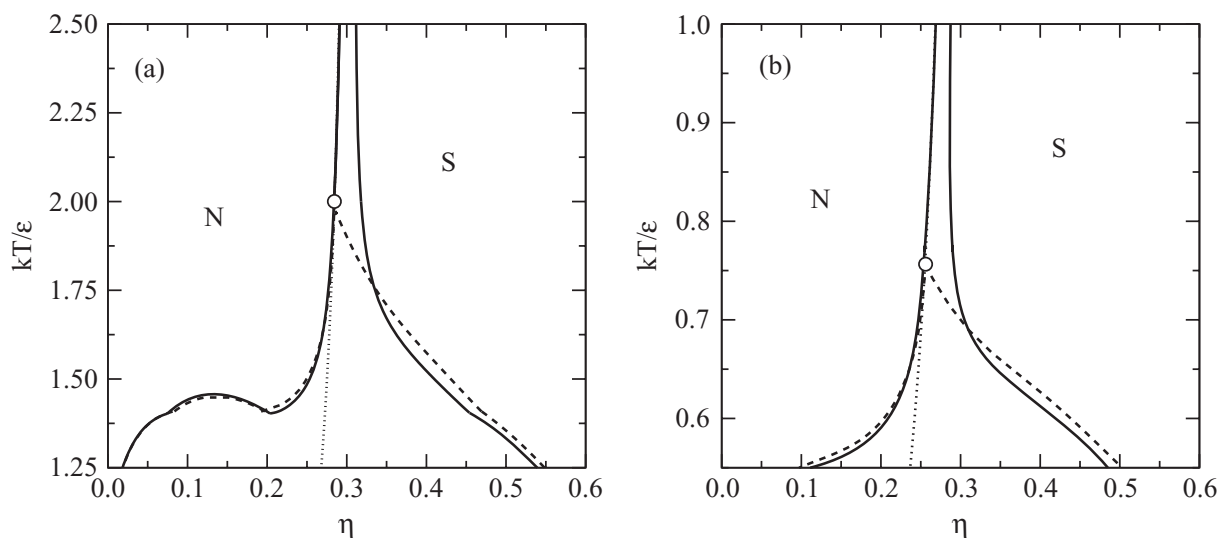

FIG. 8. Phase diagram of the polydisperse attractive Yukawa fluid. Continuous lines: coexistence boundaries for the polydisperse fluid with $\Delta=0.294$. Dashed lines: monodisperse fluid. Dotted line: nematic-smectic spinodal. (a) $\lambda=1$. (b) $\lambda=2$. 

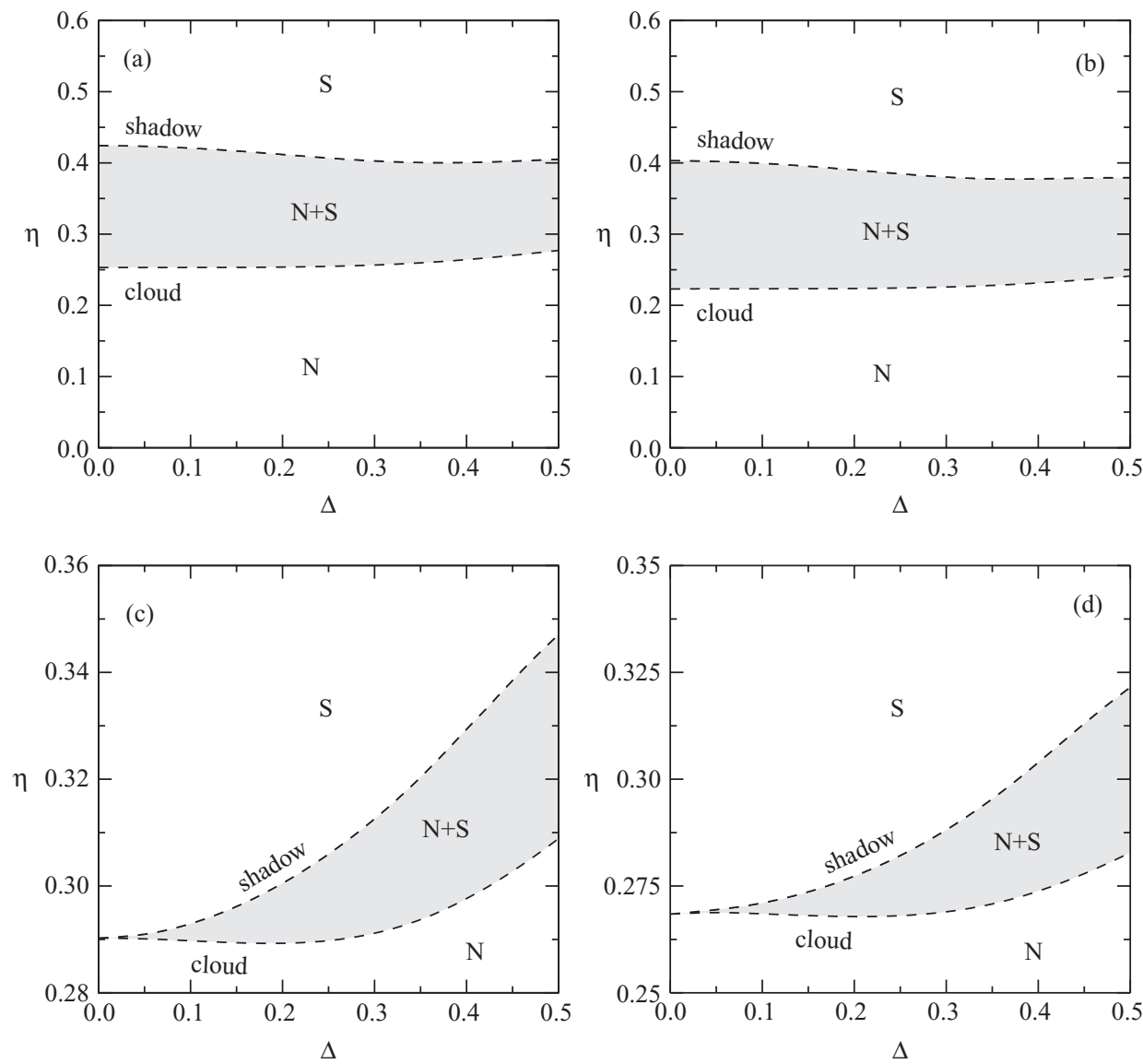

FIG. 9. Packing fraction vs polydispersity for attractive Yukawa fluid with: (a, c) $\lambda=1$; (b, d) $\lambda=2$. Temperatures are: (a) $T^{*}=1.52$, (b) 0.63 (both below corresponding tricritical points), (c) 2.50, and (d) 1.00 (both above corresponding tricritical points).

We now turn to the results. In the case of attractive forces, the NS transition can be of first order or continuous, depending on the temperature. This is shown in Fig. 6 for two values of the inverse range parameter $\lambda$. There is a tricritical point (indicated by an open circle in the figure), below which there is NS coexistence. Above the tricritical temperature the transition is continuous. This scenario is independent of the value of the interaction range. However, an interesting feature of the phase behavior is that, when the interactions are sufficiently long-ranged (i.e., $\lambda$ sufficiently small), the nematic free-energy branch presents a region of instability, indicating the existence of nematic-nematic $\left(\mathrm{N}_{1}+\mathrm{N}_{2}\right)$ demixing below a critical temperature [with an associated critical point indicated by a shaded circle in Fig. 6(a)]. Obviously, as the inverse length decreases, nematic-nematic demixing becomes even more pronounced. This is shown in Fig. 7(a), where the $\mathrm{N}_{1}+\mathrm{N}_{2}$ demixing region in the $T-\eta$ plane is represented for different values of the inverse lengths, $\lambda=0.5$, 1 , and 2. The effect of the range of the interactions on the smectic stability, which is relatively weak, can be appreciated from the change in the location of the NS spinodal as $\lambda$ is varied. As can be seen from Fig. 7(a), smectic ordering is slightly favored as the range of the interactions is increased.

The case of repulsive interactions is quite different. No nematic demixing transition exists in this case of repulsive in- teractions [Fig. 7(b)], and the NS transition is always continuous. Again the effect of varying the inverse length is weak, but different from the attractive case: for attractive forces, smectic ordering is favored as the interaction range is increased, but in the case of repulsive forces, longer-ranged interactions disfavor the formation of a layered structure with respect to a spatially uniform configuration. In any case, the effect of the interaction range on the NS transition is larger for repulsive forces.

\section{B. Polydisperse fluid}

When platelets are not equal in size, several modifications in the theory are necessary. The first is that density profiles depend on the polydispersity variable $r$, and the soft potential $V_{\text {soft }}\left(r_{\perp}, z, r, r^{\prime}\right)$ also contains a dependence via the radii of the two interacting platelets. The mean-field free-energy contribution is now

$$
\begin{aligned}
\frac{\beta \mathcal{F}_{\mathrm{mf}}[\rho]}{V}= & \frac{1}{2 d} \int_{0}^{d} d z \int_{-\infty}^{\infty} d z^{\prime} \int_{0}^{\infty} d r \int_{0}^{\infty} d r^{\prime} \\
& \times \rho(z, r) \rho\left(z^{\prime}, r^{\prime}\right) u\left(\left|z-z^{\prime}\right|, r, r^{\prime}\right),
\end{aligned}
$$

where $u\left(z, r, r^{\prime}\right)$ is defined as in Eq. (30). Next the soft interaction potential has to be specified. The soft potential will be 

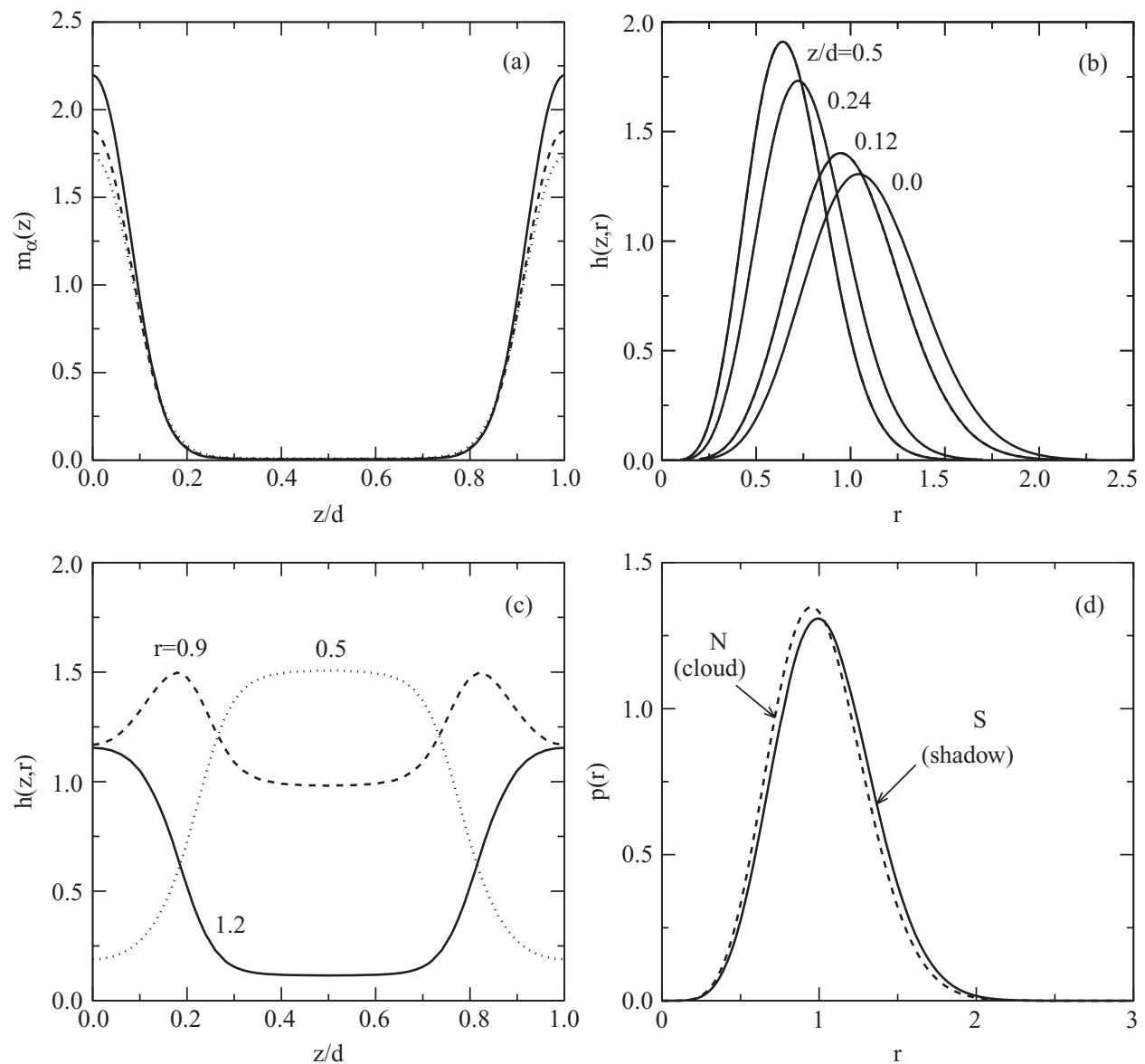

FIG. 10. Radius distribution functions in the (shadow) coexisting smectic phase for the attractive polydisperse Yukawa fluid with $\lambda=2$ at scaled temperature $T^{*}=0.6$ and degree of polydispersity $\Delta=0.294$. (a) Moments $m_{\alpha}(z)$ for $\alpha=0$ (continuous curve), 1 (dashed curve), and 2 (dotted curve). (b) Size distribution function $h(z, r)$ as a function of $r$ for different locations $z$ along one smectic period; from right to left $z / d=0,0.12,0.24$, and 0.5 (the smectic spacing is $d=1.194 L_{0}$ ). (c) Size distribution function $h(z, r)$ as a function of $z$ for different values of platelet radius; $r=1.2$ (continuous curve), 0.9 (dashed curve), and 0.5 (dotted curve). (d) Radius distribution functions $p(r)$ for the coexisting nematic (at the cloud curve) and smectic (at the shadow curve).

defined as an extension of the one used previously [Eq. (28)], but for two platelets of different radii $R=r R_{0}, R^{\prime}=r^{\prime} R_{0}$ :

$$
V_{\text {soft }}\left(r_{\perp}, z, r, r^{\prime}\right)=-\epsilon \begin{cases}\frac{e^{-\lambda\left\{\sqrt{\left(\frac{r_{\perp}}{R+R^{\prime}}\right)^{2}+\left(\frac{z}{L_{0}}\right)^{2}}-1\right\}}}{\sqrt{\left(\frac{r_{\perp}}{R+R^{\prime}}\right)^{2}+\left(\frac{z}{L_{0}}\right)^{2}},} & |z|>L_{0} \\ \text { or } & |z|<L_{0} \text { and } r_{\perp}>R+R^{\prime}\end{cases}
$$

0

otherwise.

Again, since we are now considering a polydisperse fluid, a proper calculation of the cloud-nematic and shadow-smectic curves has to be implemented. The radius distribution function used in the following contains a Gaussian tail:

$$
p(r)=C_{\nu} r^{\nu} e^{-\left(s_{\nu} r\right)^{2}},
$$

$$
C_{v}=\frac{2 s_{v}^{v+1}}{\Gamma\left(\frac{v+1}{2}\right)}, \quad s_{v}=\frac{\Gamma\left(\frac{v+2}{2}\right)}{\Gamma\left(\frac{v+1}{2}\right)} .
$$

This choice is related to the better convergence reached in the numerical implementation of Eq. (26). 

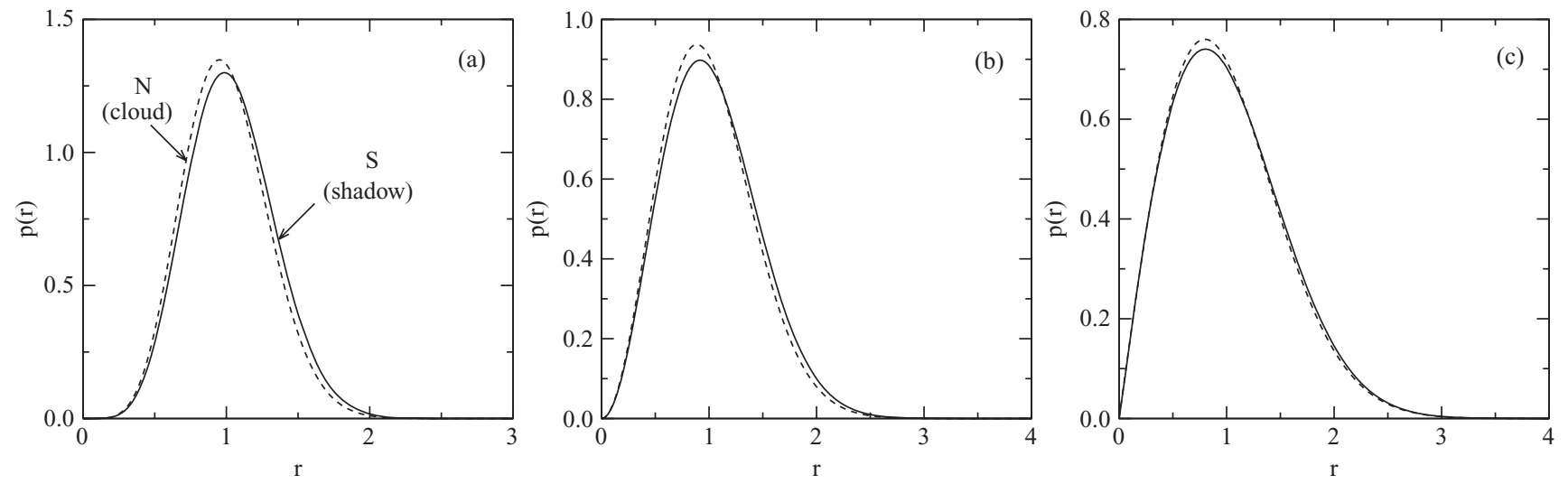

FIG. 11. Evolution of the radius distribution function $p(r)$ with increasing $\Delta$ for the nematic in the cloud curve (dashed curves) and the smectic in the shadow curve (continuous curves) at a scaled temperature $T^{*}=0.625$ and with an inverse length $\lambda=2$. (a) $\Delta=0.294$, (b) 0.422 , and (c) 0.523 .

We have performed coexistence calculations and obtained complete phase diagrams including nematic and smectic regions of stability, in this case, only for the attractive Yukawa-like potential (polydispersity is not expected to affect the phase behavior significantly in the case of repulsive forces). The phase diagrams are shown in Figs. 8(a) and 8(b), the first for an inverse length $\lambda=1$ and the second for $\lambda=2$. For the sake of comparison, the results for the monodisperse fluid have been superimposed. As far as $\mathrm{N}_{1}+\mathrm{N}_{2}$ coexistence is concerned, both the cloud and shadow curves of each nematic phase were calculated; however, their difference cannot be appreciated at the scale of the figure, so only the cloud curve is plotted (this curve chosen for consistency, as in the case of the NS coexistence only the cloud-nematicshadow-smectic curve was computed). The most important change brought about by polydispersity is that the tricritical point moves to very high values even for small values of $\Delta$, and therefore the NS phase transition becomes of first order in the reasonable range of temperatures. Again, for the longer-ranged potential, there exists a region of nematic demixing that is missing for shorter-ranged interactions, a feature not much affected by polydispersity.

To show the effect of polydispersity on the NS transition in attractive platelets and ascertain how the nature of the transition changes from the monodisperse to the polydisperse case, we plot in Fig. 9 the coexistence packing fraction of the cloud-nematic and shadow-smectic curves as a function of polydispersity for $\lambda=1$ and 2 . The effect is weak when the temperature is below the tricritical point of the monodisperse case, since the density gap hardly changes with $\Delta$. By contrast, for temperatures above the tricritical-point temperature of the monodisperse fluid, and as the polydispersity is increased from $\Delta=0$, the density gap opens up quite rapidly, meaning that the transition changes from second to first order even for a low degree of polydispersity. This is shown in Figs. 9(c) and 9(d).

We have also studied the effect of microsegregation, i.e., of the spatial redistribution of particles with different sizes occurring along one period in the smectic phase in the coexisting shadow-smectic phase. For this purpose, the moment distribution profiles $m_{\alpha}(z)$, defined previously, are useful. These functions are plotted in Fig. 10(a), together with the distribution function $h(z, r)$ as a function of $r$ for fixed $z$ in
Fig. 10(b), and as a function of $z$ for fixed $r$ in Fig. 10(c), all for $\Delta=0.294$ and $T^{*}=0.6$. The radius distribution functions $p(r)$ in the $\mathrm{N}$ (cloud) and $\mathrm{S}$ (shadow) phases are shown in Fig. 10(d). The functions $m_{\alpha}(z)$ reflect the fact that platelets are arranged in layers, with larger-sized platelets lying exactly at the layers and with platelets being progressively smaller as one moves into the interstitial region. This microsegregation is more clearly appreciated in Fig. 10(b), where the size distribution function $h(z, r)$ is plotted for selected values of the $z$ coordinate between the location of the layer $z=0$ and the intermediate distance $z=d / 2$. At the layers, the maximum of the distribution occurs for larger values of the radius, whereas at the interstitial the maximum is located at a rather low radius. The width of the distribution decreases from the layer to the interstitial. The shift in average size as one moves along a smectic period is again visible in Fig. 10(c): of the three sizes considered, larger particles $(r=1.2)$ peak at the layers, and smaller ones at the interstitials, with intermediate sizes in intermediate positions.

The size distribution functions of nematic and smectic phases, Fig. 11, are quite similar, with a slightly larger composition of the larger-sized platelets in the smectic phase, but with almost identical widths. This trend is less and less apparent as the polydispersity $\Delta$ is increased: nematic and smectic distributions become more and more similar, with mean radii that shift to lower values, widths that become broader, and tails that decay more slowly.

\section{CONCLUSIONS}

In this work we have discussed the phase behavior of suspensions made of platelets. We were motivated by the recent finding of smectic ordering in an aqueous suspension of $\alpha-\mathrm{ZrP}$-based platelets of the same thickness but very polydisperse in diameter. The work has focused on the layered arrangement (smectic phase) of platelets arising from a suspension of positionally disordered, but perfectly orientationally ordered, particles (nematic phase). The high polydispersity in diameter prevents the formation of the columnar phase, which has not been considered in the present work. The assumption of parallel, perfectly oriented platelets should be valid when the aspect (diameter-to-thickness) ratio is very high, which is the case in the $\alpha-\mathrm{ZrP}$ samples and in 
many other discotic systems made from mineral materials. This assumption allows us to use the powerful fundamentalmeasure density-functional theory for hard platelets, which is expected to accurately describe ordered arrangement of platelets, due to the emphasis of the theory on spatial correlations. Our first aim was to obtain a picture of how the degree of diameter polydispersity affects the nematic-smectic phase transition. Polydispersity tends to stabilize the smectic phase, due to a microsegregation effect which allows larger and similar particles to populate the layers and pack more efficiently. The platelet volume fraction of the sample at the nematic-smectic transition can be reduced by $5 \%$ for a degree of diameter polydispersity of $30 \%$, quite typical of the experimental system. The transition is found to be always continuous, regardless of the degree of polydispersity.

Our second aim was to explore the consequences of soft interactions on the nematic-smectic transition. Suspensions of platelets can be obtained using different techniques, and both attractive and repulsive particles can be designed. We have contemplated both cases using a simple monotonic Yukawa-like interaction potential function which can be positive or negative. The sign of the soft interactions profoundly affects the phase behavior. For example, when platelets are identical (zero polydispersity), soft repulsive forces always induce a continuous nematic-smectic transition, with the smectic phase stabilizing with respect to the nematic for a decreasing range of the force. By contrast, when the interactions are attractive, the transition becomes of the first order for temperatures below a tricritical temperature. In addition, when the interaction range is sufficiently long, there appears a nematic demixing phenomenon, whereby two nematic phases of different platelet concentration appear in the suspension. Both nematic phases may coexist with a smectic phase at a triplepoint temperature. In this case the smectic phase is slightly destabilized as the range of the force is decreased. Polydispersity changes this scenario to some extent. While nematic demixing is not much affected (at least for polydispersities $\lesssim 30 \%$ ), the tricritical temperature moves to very high values, and the nematic-smectic transition becomes of first order even for a small degree of polydispersity.

The application of the present results to real experimental samples may be done after cautiously considering the type of interactions present in the platelets. Surface charges, counterions, hydration effects, etc., may play a role in the total balance between attractive and repulsive forces. Also, the interactions may have different sign with respect to distance. Another aspect, not contemplated in the present work, is the role of the isotropic phase, which is stable at lower packing fractions. The analysis of the isotropic phase requires a different theoretical framework in order to account for orientational degrees of freedom. For example, the fundamental-measure density functional for freely rotating hard platelets with vanishing thickness ${ }^{39}$ (adequately extended to the polydisperse case) could be a good candidate for a reference system in perturbation theories for soft platelets. A complete picture would be obtained by incorporating the columnar phase, which could in principle be done within the present theory, but at a higher computational cost. The computation of the full phase diagram, and the study of how polydispersity in diameter and/or thickness, soft direct and solvent-induced interactions, etc., change the phase behavior of platelet suspensions, is a big challenge from a theoretical point of view. This work represents a step toward that goal.

\section{ACKNOWLEDGMENTS}

We greatly appreciate enlightening discussions with Dr. Z. Cheng. This work has been partly financed by grants NANOFLUID, MOSAICO, and MODELICO from Comunidad Autónoma de Madrid (Spain), and Grant Nos. FIS2007-65869-C03-01, FIS2008-05865-C02-02, FIS201022047-C05-01, and FIS2010-22047-C05-04 from Ministerio de Educación y Ciencia (Spain).

${ }^{1}$ H. Z. Zocher, Z. Anorg. Allg. Chem. 147, 91 (1925).

${ }^{2}$ I. Langmuir, J. Chem. Phys. 6, 873 (1938).

${ }^{3}$ J. D. Bernal and I. Fankuchen, J. Gen. Physiol. 25, 111 (1941).

${ }^{4}$ L. Onsager, Ann. N. Y. Acad. Sci. 51, 627 (1949).

${ }^{5}$ M. P. B. van Bruggen, F. M. van der Kooij, and H. N. W. Lekkerkerker, J. Phys.: Condens. Matter 8, 9451 (1996).

${ }^{6}$ F. M. van der Kooij and H. N. W. Lekkerkerker, J. Phys. Chem. B 102, 7829 (1998).

${ }^{7}$ A. B. D. Brown, S. M. Clarke, and A. R. Rennie, Langmuir 14, 3129 (1998)

${ }^{8}$ J. A. C. Veerman and D. Frenkel, Phys. Rev. A 41, 3237 (1990).

${ }^{9}$ H. N.W. Lekkerkerker, P. Coulon, R. van der Hagen, and R. Deblieck, J. Chem. Phys. 80, 3427 (1984).

${ }^{10}$ M. A. Bates and D. Frenkel, J. Chem. Phys. 109, 6193 (1998).

${ }^{11} \mathrm{G}$. Cinacchi, L. Mederos, and E. Velasco, J. Chem. Phys. 121, 3854 (2004).

${ }^{12}$ A. Mourchid, A. Delville, J. Lambard, E. Lecolier, and P. Levitz, Langmuir 11, 1942 (1995).

${ }^{13}$ D. van der Beek and H. N. W. Lekkerkerker, Langmuir 20, 8582 (2004).

${ }^{14}$ F. M. van der Kooij, K. Kassapidou, and H. N. W. Lekkerkerker, Nature (London) 406, 868 (2000).

${ }^{15}$ F. M. van der Kooij, M. Vogel, and H. N. W. Lekkerkerker, Phys. Rev. E 62, 5397 (2000)

${ }^{16}$ J. C.P. Gabriel, F. Camerel, B. J. Lemaire, H. Desvaux, P. Davidson, and P. Batail, Nature (London) 413, 504 (2001).

${ }^{17}$ N. Wang, S. Liu, J. Zhang, Z. Wu, J. Chen, and D. Sun, Soft Matter 1, 428 (2005)

${ }^{18}$ D. Sun, H.-J. Sue, Z. Cheng, Y. Martínez-Ratón, and E. Velasco, Phys. Rev. E 80, 041704 (2009).

${ }^{19}$ S. Asakura and F. J. Oosawa, J. Polym. Sci. 33, 183 (1958).

${ }^{20}$ S. D. Zhang, P. A. Reynolds, and J. S. van Duijneveldt, J. Chem. Phys. 117, 9947 (2002).

${ }^{21}$ H. H. Wensink and H. N. W. Lekkerkerker, Europhys. Lett. 66, 125 (2004).

${ }^{22}$ L. Luan, W. Li, S. Liu, and D. Sun, Langmuir 25, 6349 (2009).

${ }^{23}$ A. V. Petukhov, D. van der Beek, R. P. A. Dullens, I. P. Dolbnya, G. J. Vroege, and H. N. W. Lekkerkerker, Phys. Rev. Lett. 95, 077801 (2005).

${ }^{24}$ L. Y. Sun, W. J. Boo, D. H. Sun, A. Clearfield, and H. J. Sue, Chem. Mater. 19, 1749 (2007)

${ }^{25}$ H. N. Kim, S. W. Keller, T. E. Mallouk, J. Schmitt, and G. Decher, Chem. Mater. 9, 1414 (1997).

${ }^{26}$ M. Schmidt and A. R. Denton, Phys. Rev. E 65, 021508 (2002).

${ }^{27}$ S. V. Savenko and M. Dijkstra, J. Chem. Phys. 124, 234902 (2006).

${ }^{28}$ M. A. Bates and D. Frenkel, Phys. Rev. E 62, 5225 (2000).

${ }^{29}$ N. Clarke, J. A. Cuesta, and R. Sear, J. Chem. Phys. 113, 5817 (2000).

${ }^{30}$ A. Speranza and P. Sollich, J. Chem. Phys. 117, 5421 (2002).

${ }^{31}$ A. Speranza and P. Sollich, Phys. Rev. E 67, 061702 (2003).

${ }^{32}$ Y. Martínez-Ratón and J. A. Cuesta, Phys. Rev. Lett. 89, 185701 (2002).

${ }^{33}$ Y. Martínez-Ratón and J. A. Cuesta, J. Chem. Phys. 118, 10164 (2003).

${ }^{34}$ S. Varga, A. Galindo, and G. Jackson, Phys. Rev. E 66, 011707 (2002).

${ }^{35}$ L. Harnau, D. Rowan, and J.-P. Hansen, J. Chem. Phys. 117, 11359 (2002).

${ }^{36}$ M. Bier, L. Harnau, and S. Dietrich, Phys. Rev. E 69, 021506 (2004).

${ }^{37}$ Y. Martínez-Ratón, J. A. Capitán, and J. A. Cuesta, Phys. Rev. E 77, 051205 (2008).

${ }^{38}$ J. A. Capitán, Y. Martínez-Ratón, and J. A. Cuesta, J. Chem. Phys. 128, 194901 (2008)

${ }^{39}$ A. Esztermann, H. Reich, and M. Schmidt, Phys. Rev. E 73, 011409 (2006). 